\title{
Wavevector spectral signature of decay instability in space plasmas
}

\author{
Horia Comişel $^{1,2}$, Yasuhito Narita $^{3}$, and Uwe Motschmann ${ }^{1,4}$ \\ ${ }^{1}$ Institut für Theoretische Physik, Technische Universität Braunschweig, Mendelssohnstr. 3, 38106 Braunschweig, Germany \\ ${ }^{2}$ Institute for Space Sciences, Atomiştilor 409, P.O. Box MG-23, Bucharest-Măgurele, 077125, Romania \\ ${ }^{3}$ Space Research Institute, Austrian Academy of Sciences, Schmiedlstr. 6, 8042 Graz, Austria \\ ${ }^{4}$ Deutsches Zentrum für Luft- und Raumfahrt, Institut für Planetenforschung, Rutherfordstr. 2, 12489 Berlin, Germany
}

Correspondence: Horia Comişel (h.comisel@tu-braunschweig.de)

Received: 16 April 2020 - Discussion started: 18 May 2020

Revised: 30 July 2020 - Accepted: 18 January 2021 - Published: 22 February 2021

\begin{abstract}
Identification of a large-amplitude Alfvén wave decaying into a pair of ion-acoustic and daughter Alfvén waves is one of the major goals in the observational studies of space plasma nonlinearity. In this study, the decay instability is analytically evaluated in the 2-D wavenumber domain spanning the parallel and perpendicular directions to the mean magnetic field. The growth-rate determination of the density perturbations is based on the Hall MHD (magnetohydrodynamic) wave-wave coupling theory for circularly polarized Alfvén waves. The diagrams of the growth rates versus the wavenumber and propagation angle derived in analytical studies are replaced by 2-D wavenumber distributions and compared with the corresponding wavevector spectrum of density and magnetic field fluctuations. The actual study reveals a perpendicular spectral pattern consistent with the result of a previous study based on 3-D hybrid numerical simulations. The wavevector signature of the decay instability observed in the two-dimensional wavenumber domain ceases at values of plasma beta larger than $\beta=0.1$. Growth-rate maps serve as a useful tool for predictions of the wavevector spectrum of density or magnetic field fluctuations in various scenarios for the wave-wave coupling processes developing at different stages in space plasma turbulence.
\end{abstract}

\section{Introduction}

Parametric instabilities driven by large-amplitude Alfvén waves have extensively been investigated by analytical studies or numerical simulations in one- or multidimensional approaches. A systematic analytical analysis of the multidi- mensional features of the parametric instabilities has been initiated by Viñas and Goldstein (1991a, b) by applying the Hall magnetohydrodynamic (hereafter, Hall-MHD) theory to a large-amplitude field-aligned Alfvén wave with lefthand and right-hand circular polarization. Results of the twodimensional predictions of Viñas and Goldstein (1991a, b) have successfully been confirmed by later numerical simulations. Obliquely propagating daughter waves excited by the decay of a field-aligned Alfvén wave have been observed in 2-D MHD numerical simulations by Ghosh et al. (1993) for a low-beta regime. Other studies on the nonlinear interaction of obliquely propagating Alfvén waves confirm that the growth rate of the decay instability in direction oblique to the mean magnetic field is typically smaller than the fieldaligned decay; see, for example, Mjølhus and Hada (1990), Laveder et al. (2002), and Nariyuki et al. (2008). By using two-dimensional hybrid simulations, Matteini et al. (2010) discovered that a broad spectrum of Alfvén and density fluctuations is developing perpendicular to the direction of the mean magnetic field at the decay of a linear polarized Alfvén pump wave with oblique direction of propagation in low-beta plasmas. Gao et al. (2013) reported by means of 2-D hybrid simulations that a linear polarized Alfvén pump wave with parallel propagation can also generate a perpendicular spectrum of daughter waves. Comişel et al. (2019) observed recently a perpendicular spectrum of daughter waves by using field-aligned Alfvén pump waves with circular left-hand polarization and 3-D hybrid simulations. This result was not predicted by previous 2-D numerical simulations. The threedimensional setup was also used by Comişel et al. (2020) for analyzing the evolution of large-amplitude Alfvén waves into 
the azimuthal (or transversal) plane with respect to the mean magnetic field in low-beta plasmas.

The purpose of this study is to recall the former HallMHD analytic approach developed by Viñas and Goldstein (1991a). The analytical predictions show that at very low beta values, the oblique decay of a circularly polarized Alfvén wave becomes competitive with the field-aligned decay. We are looking at whether the solutions of the dispersion equation provided by Viñas and Goldstein (1991a) model can or cannot drive a perpendicular spectrum of daughter waves in accordance with the prediction of the 3-D hybrid simulation. In the two-dimensional analytical analysis, the dispersion equation is typically solved by setting a priori the propagation direction of the daughter wave, and the complex solution for the frequency is investigated in the wavenumber domain. Here we solve and display the imaginary part of frequencies (namely, growth rates) of the dispersion equation into a wavevector-spectrum-like diagram along the parallel and perpendicular directions to the mean magnetic field. On the basis of this study, the developed perpendicular spectrum of daughter waves can be considered a signature for the decay of a left-handed circularly polarized Alfvén pump wave in low-beta plasmas. This pattern describing the oblique-decay process is vanishing for larger values of plasma beta parameter.

In the two-dimensional analysis of Viñas and Goldstein (1991a), the authors concluded that at moderate oblique propagation angles of the daughter waves the oblique-decay growth rates decrease and split into narrow band-profiles (in the wavenumber domain). In the actual approach, we explore in more detail the growth rates at beta values much smaller than the typical value $(\beta=0.5)$ used in the previous study. The obtained solutions of the dispersion equations represented in a parallel and perpendicular wavenumber diagram reveal new spectral features of the decay instability in lowbeta plasmas which have never been pointed out in former analytical investigations. Furthermore, this result strengthens the idea of multichannel coupling of decay instability as a proof of concept proposed by Comişel et al. (2019) in a former study based on numerical 3-D hybrid simulations. By following the Hall-MHD formalism of Viñas and Goldstein (1991a), we constructed the $k_{\|}-k_{\perp}$ spectrum, and the results are in good agreement between the numerical simulation and the analytic treatment.

\section{Method and results}

We use the analytical analysis developed by Viñas and Goldstein (1991a, b) based on the two-fluid plasma model together with the generalized Ohm's law. The dispersive effects are driven by the ion inertia and the Hall term. Monochromatic parallel-propagating Alfvén waves are exact solutions of the nonlinear MHD equations describing a plasma system. Starting from this property, the set of equations for the
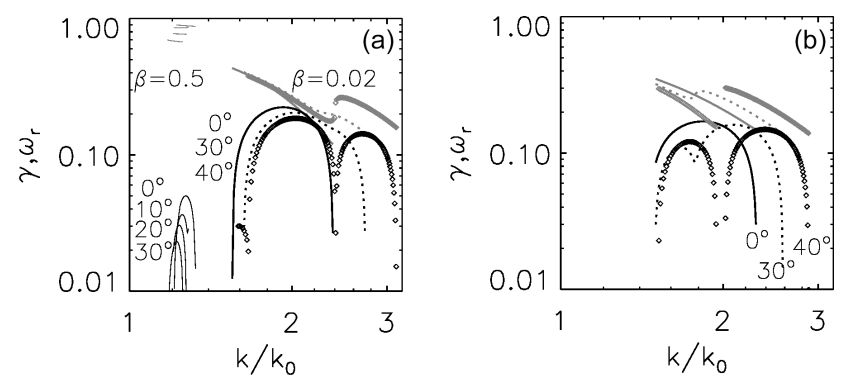

Figure 1. Solutions of the dispersion equation (growth rates in black and frequencies in gray) versus normalized wavenumber for righthanded (a) and left-handed (b) polarized Alfvén pump waves at propagation angles 0,30 , and $40^{\circ}$ and plasma $\beta=0.02$. (a) also shows the result obtained for right-hand polarization and plasma $\beta=0.5$.

evolution of density, flow velocity, and magnetic field is linearized by using a perturbation expansion in order to define a linear-mode wave (or eigenmode of the system) around the equilibrium of each of the above mentioned quantities. Each linear mode is specified by its frequency and wavevector. The wave-wave coupling of the large-amplitude Alfvén pump wave with a density perturbation of wavevector $\boldsymbol{k}$ and frequency $\omega$ is conducting to side-band daughter waves expressed by the relations $\boldsymbol{k}^{ \pm}=\boldsymbol{k} \pm \boldsymbol{k}_{\mathbf{0}}$ and $\omega^{ \pm}=\omega \pm \omega_{0}$, where $\boldsymbol{k}^{ \pm}$and $\omega^{ \pm}$describe the wavevectors and frequencies of the daughter waves, respectively; $\boldsymbol{k}_{0}=k_{0} \boldsymbol{e}_{\|}$, where $k_{0}$ is the wavenumber of the Alfvén pump wave; $\boldsymbol{e}_{\|}$is the unity vector parallel to the mean magnetic field; and $\omega_{0}$ is the frequency of the Alfvén pump wave. The daughter waves are allowed to propagate parallel and obliquely to the magnetic field. The general dispersion equation is derived in terms of $6 \times 6$ matrices and depends on six independent parameters: frequency, wavenumber and angle of propagation of the linear mode, amplitude and wavenumber of the pump wave, and plasma $\beta$ parameter. The frequency and growth rate are normalized according to Viñas and Goldstein (1991a) as $\omega_{r}=\operatorname{Re}(\omega) / k_{0} V_{\mathrm{A}}$ and $\gamma=\operatorname{Im}(\omega) / k_{0} V_{\mathrm{A}}$, where $V_{\mathrm{A}}$ is Alfvén speed. The plasma beta is defined as $\beta=V_{\mathrm{s}}^{2} / V_{\mathrm{A}}^{2}$ with $V_{\mathrm{s}}$ being the sound speed.

The dispersion equation is implemented and solved by using the Mathematica software. We first investigate the solutions $\left(\omega_{r}, \gamma\right)$ for the decay of a right-handed polarized Alfvén wave with the same parameters used by Viñas and Goldstein (1991b). Figure 1 (left panel) reports the growth rates (solid line) and frequencies (gray solid line) obtained for plasma $\beta=0.5$. The amplitude of the Alfvén pump wave is 0.2 (normalized to the background magnetic field), and its wavenumber (normalized in terms of ion inertial length) $k_{0} V_{\mathrm{A}} / \Omega_{\mathrm{p}}$ is 0.3 , where $\Omega_{\mathrm{p}}$ is the ion gyrofrequency (for protons). The maximum growth rate for parallel propagation is obtained at $k / k_{0} \approx 1.25$. The peaks of the growth rates determined at oblique-propagation angles of 10,20 , and $30^{\circ}$ are 

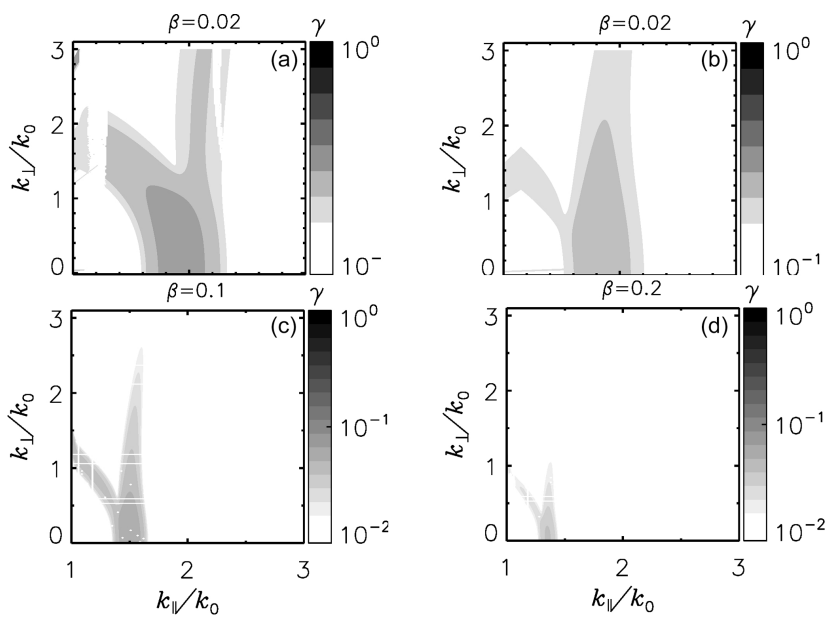

trum of density or magnetic field fluctuations. The mapping of the growth rates in such coordinates is helpful in our study for a twofold purpose discussed below: (i) establishing or finding of a specific pattern for the parametric decay in the wavevector domain and (ii) direct comparison of the analytical predictions with spectra of fluctuations obtained from numerical simulations (or presumably in situ measurements).

The top panels of Fig. 2 are the arrangement of the plots shown in Fig. 1 into the parallel and perpendicular wavenumber domain for the right-handed and left-handed Alfvén pump waves. The growth rates in the new coordinates are obtained by solving the dispersion relation for $k / k_{0}$ wavenumbers spanning the domain $(1,3)$ and $(0,3)$ along the parallel and perpendicular directions with respect to the main magnetic field. Because this procedure is a demanding numerical task, the solutions to the dispersion equations are searched in a limited wavenumber range where decay instability is expected (for instance, the domain below $k_{\|} / k_{0}=1$, where modulational instability could be operational, is omitted). The solution is determined for a given pair of values $\left(k_{\|}, k_{\perp}\right)$ which is then advanced by a discrete $\Delta k$ step for each parallel or perpendicular direction. The resulting solutions are smoothed and represented in the wavevector domain. One may observe an arc-shaped branch of solutions and a perpendicular one for the right-handed polarized Alfvén pump wave. For left-handed polarized waves, the perpendicular branch clearly dominates the arc-shaped branch in accordance with the profiles drawn in Fig. 1. The bottom panels of Fig. 2 present the map of growth rates determined at larger beta values for left-handed polarized Alfvén pump waves. While the parallel wavenumber of the maximum growth rate is shifting to lower values, the perpendicular branch becomes weaker, and at larger beta values $(\beta>0.1)$ the oblique decay becomes insignificant with respect to the lowest-beta analyzed case. From this analysis, we conclude that a perpendicular spectral pattern of the decay products can be associated with the decay process of a circularly polarized Alfvén wave in low-beta plasma.

In Fig. 3 we compare the result of the actual study for the left-handed polarization and beta value of 0.02 with the 2-D wavenumber spectrum of density and magnetic field fluctuations from a former 3-D hybrid simulation (Comişel et al., 2019). First, the growth-rate map given in Fig. 2 is extended towards both positive and negative perpendicular wavenumbers. Second, the growth rates for the lower side-band daughter waves $\left(\omega_{-}=\omega-\omega_{0}\right)$ describing Alfvén waves with backward propagation are added at (negative) wavenumbers $k_{\|}^{-}=$ $k_{\|}-k_{0}$ on the basis that $\operatorname{Im}\left(\omega_{-}\right) \equiv \operatorname{Im}(\omega)$ according to the wave-wave coupling scheme. The magnetic field and density fluctuations given in the right panel of Fig. 3 are obtained from a former 3-D hybrid simulation based on AIKEF code (Müller et al., 2011) and a similar scenario with the current study. Magnetic field fluctuations are represented at negative wavenumbers corresponding to the backward propagating Alfvén daughter waves, while the compressional for- 

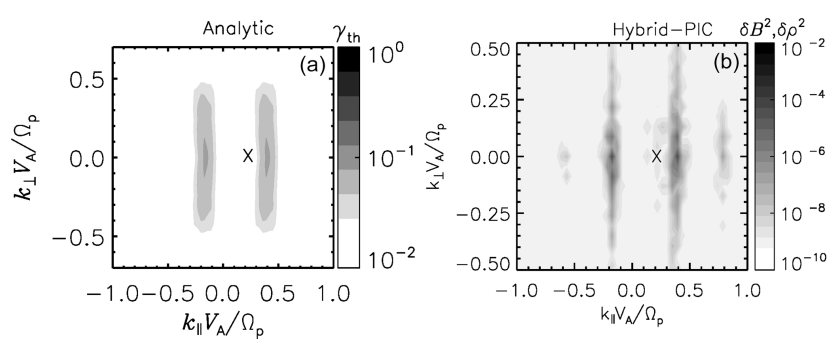

Figure 3. Comparison between the extended map of the growth rates in the $k_{\|}-k_{\perp}$ wavenumber domain (a) and 2-D wavenumber spectrum of density and magnetic field field fluctuations (b) from Comişel et al. (2019). The location of Alfvén pump waves is marked by a cross symbol.

ward propagating waves (acoustic-like) are shown as density fluctuations along the positive wavenumbers. Furthermore, the 2-D wavenumber spectra are filtered in frequency domain such that only the frequencies expected for the Alfvén daughter waves at $\omega=\omega_{s}-\omega_{0}$ and for density mode at $\omega=\omega_{s}$ (here $\omega_{s}$ is the frequency of the ion acoustic wave) are shown. The spectral analyses report the Alfvén daughter modes at $k_{\|} V_{\mathrm{A}} / \Omega_{\mathrm{p}}$ of $\sim-0.2$ and the sound daughter waves at $k_{\|} V_{\mathrm{A}} / \Omega_{\mathrm{p}}$ of $\sim 0.4$. There is a good qualitative match between the two panels. As we already mentioned, the analytical model does not consider the wave damping or the harmonics of the excited daughter modes. The actual study and former hybrid simulation suggest that the field-aligned decay is accompanied by an oblique-decay process developing a perpendicular spectrum of density and magnetic field fluctuations.

\section{Discussion}

Former studies based on 2-D hybrid simulations, e.g., Matteini et al. (2010), reported a transversal spectrum of daughter waves observed at the decay of a large-amplitude Alfvén wave with linear polarization in low-beta plasmas. Matteini et al. (2010) discussed the observed result in terms of the finite oblique angle of propagation of the imposed Alfvén pump wave with respect to the mean magnetic field. Gao et al. (2013) confirmed the former 2-D numerical study and even noticed a similar perpendicular spectrum of waves excited at the decay of a field-aligned pump wave. Two decades before, the two-dimensional analytical studies of Viñas and Goldstein (1991b) suggest, on the basis of the beta dependence of the oblique-decay growth rates, that the decay process of a field-aligned circularly polarized Alfvén wave might become important at finite angles of propagation for the daughter waves in low-beta plasmas. The mechanism controlling the oblique decay instabilities has been discussed in relation with the coupling between the electrostatic (dominant at parallel propagation) and electromagnetic (dominant at high oblique angles) terms in the Hall-MHD nonlinear equations. By representing the growth rates provided by the Viñas and Goldstein (1991a) formalism in the wavevector domain, the perpendicular spectrum of daughter waves can be straightforwardly noticed in Fig. 2, while this feature is not obvious in the usual representation in Fig. 1. The perpendicular decay of daughter waves has been therefore predicted by the Hall-MHD theory well before the first observations provided by 2-D hybrid simulations in low-beta plasmas. On the basis of this result, one may expect that growthrate maps in the wavevector domain could reveal new properties of parametric instabilities which can be further investigated or diagnosed by analytical treatments or by numerical simulations.

There are still open questions started in early studies about the role played by parametric instabilities to the generation of turbulent cascades (Hoshino and Goldstein, 1989; Ghosh and Goldstein, 1994). The perpendicular spectrum of daughter waves triggered by the nonlinear wave-wave coupling processes is superposed to the perpendicular spectrum of plasma turbulence generated by the post-saturation processes of the decay instability. The plasma anisotropy index as a quantitative measure of the directional (perpendicular) turbulence evolution describes the wavevector anisotropy of a two-dimensional spectrum of magnetic fluctuations (Shebalin et al., 1983). An increasing number of 2-D or even 3-D wavevector spectra are observationally being reported from in situ solar wind measurements; see e.g., Narita et al. (2010); Narita (2014), and Narita et al. (2014). It would be interesting to compare the beta dependence of the wavevector anisotropy of solar wind turbulence, e.g., Comişel et al. (2014), with the anisotropy determined from the growth-rate maps of decay instability in its dependence on plasma beta parameter as they are displayed in Fig. 2.

To our knowledge, the current study is the first step to bring together the results of the Hall-MHD analytic approach and kinetic simulations, in emphasizing the generation of oblique daughter waves through their nonlinear evolution described by the MHD model. One may also notice that the MHD description can be helpful to clarify observed properties of the parametric instabilities which could originate in the kinetic approach by nonphysical fluctuations due to the particle discreteness expected in hybrid or particle-in-cell simulations.

As limitations of the Viñas and Goldstein (1991a) method used in this analysis, the linear dispersion was restricted to excitation of only fundamental side-band daughter waves (i.e., no harmonics are allowed). The electron-inertia effect is neglected; one may expect that timescales or length of interest is larger than the electron gyroperiod or electron inertial length, respectively. The validity of the MHD approach with included Hall term is thus limited up to higher frequencies close to the ion gyrofrequency. The Landau and cyclotron damping effects have also been neglected in the MHD approach. 


\section{Summary}

In conclusion, the analytic method developed by Viñas and Goldstein (1991a) prescribes that, in low-beta plasmas, circularly polarized Alfvén waves decay into parallel and obliquely propagating daughter waves. The growth-rate values of the decay process plotted in the two-dimensional domain of the wavevector parallel and perpendicular to the mean magnetic field evince a displacement of the solutions into two branches: a perpendicular one predominant for both left- and right-hand polarization and an arc-shaped one which is stronger for the right-hand polarization. The oblique decay significantly decreases at beta values larger than $\beta>$ 0.1 . The theoretical prediction for the left-handed polarized Alfvén pump wave derived in the 2-D wavenumber domain is consistent with the 2-D spectrum of density and magnetic field fluctuations resulting from 3-D hybrid simulations.

Growth-rate maps as those discussed above can be conveniently obtained for various values of the input parameters describing the dispersion equation. A catalogue of maps realized by a systematic analysis for plasma beta, amplitude of pump wave, polarization, or amount of dispersion can provide valuable information for further investigations of parametric instabilities by using hybrid or full-particle numerical simulations in two- or three-dimensional approaches. The growth-rate maps derived by analytical models and subsequently confirmed by numerical simulations can be helpful in future studies as predictions for the spectrum of density or magnetic field fluctuations expected from in situ measurements. Furthermore, the particular signature of the oblique decay can serve as a piece of evidence of wave-wave coupling processes acting at different evolution stages in space plasma turbulence.

Code and data availability. Software code and data sets are available by writing to the following email addresses: h.comisel@tubraunschweig.de or comisel@spacescience.ro.

Author contributions. HC worked on theory implementation and article writing. YN worked on representation of wave nonlinearities and application to space plasmas. UM worked on discussion and supervised the study. All authors read and approved the final article.

Competing interests. The authors declare that they have no conflict of interest.

Acknowledgements. Yasuhito Narita is grateful to Masahiro Hoshino and staff in his group at The University of Tokyo for their hospitality and discussion during the research visit, which was supported by the Japan Society for the Promotion of Science, Invitational Fellowship for Research in Japan (Long-term) under grant
FY2019 L19527. We also acknowledge support by the German Research Foundation and the Open Access Publication Funds of the Technical University of Braunschweig.

Financial support. This research has been supported by the Deutsche Forschungsgemeinschaft (grant no. MO539/20-1). The work conducted by Horia Comisel in Bucharest is supported by ESA project MAGICS, PRODEX,contract C4000127660.

This open-access publication was funded by Technische Universität Braunschweig.

Review statement. This paper was edited by Dalia Buresova and reviewed by one anonymous referee.

\section{References}

Comişel, H., Narita, Y., and Motschmann, U.: Wavevector anisotropy of plasma turbulence at ion kinetic scales: solar wind observations and hybrid simulations, Nonlin. Processes Geophys., 21, 1075-1083, https://doi.org/10.5194/npg-21-10752014, 2014.

Comişel, H., Narita, Y., and Motschmann, U.: Multi-channel coupling of decay instability in three-dimensional low-beta plasma, Ann. Geophys., 37, 835-842, https://doi.org/10.5194/angeo-37835-2019, 2019.

Comişel, H., Narita, Y., and Motschmann, U.: Alfvén wave evolution into magnetic filaments in 3-D space plasma, Earth Planet. Space, 72, 32, https://doi.org/10.1186/s40623-02001156-8, 2020.

Gao, X., Lu, Q., Li, X., Shan, L., and Wang., S.: Parametric instability of a monochromatic Alfven wave: Perpendicular decay in low beta plasma, Phys. Plasmas, 20, 072902, https://doi.org/10.1063/1.4816703, 2013.

Ghosh, S. and Goldstein, M. L.: Nonlinear evolution of a largeamplitude circularly polarized Alfvén wave: Low beta, J. Geophys. Res., 99, 13352-13362, 1994.

Ghosh, S., Viñas, A. F., and Goldstein, M. L.: Parametric instabilities of a large-amplitude circularly polarized Alfvén wave: Linear growth in two-dimensional geometries, J. Geophys. Res., 98, 15561-15570, https://doi.org/10.1029/93JA01534, 1993.

Hoshino, H. and Goldstein, M. L.: Time evolution from linear to nonlinear stages in magnetohydrodynamic parametric instabilities, Phys. Fluids B-Plasma, 1, 1405, https://doi.org/10.1063/1.858971, 1989.

Laveder, D., Passot, T., and Sulem, P.L.: Transverse dynamics of dispersive Alfvén waves. II. Driving of a reduced magnetohydrodymanic flow, Phys. Plasmas, 9, 305-314, https://doi.org/10.1063/1.1417511, 2002.

Matteini, L., Landi, S., Del Zanna, L., Velli, M., Hellinger, P.: Parametric decay of linearly polarized shear Alfvén waves in oblique propagation: One and two-dimensional hybrid simulations, Geophys. Res. Lett., 37, L20101, https://doi.org/10.1029/2010GL044806, 2010. 
Mjølhus, E. and Hada, T.: Oblique stability of circularly polarized MHD waves, J. Plasma Phys., 43, 257-268, https://doi.org/10.1017/S002237780001477X, 1990.

Müller, J., Simon, S., Motschmann, U., Schüle, J., Glassmeier, K. H. and Pringle, G. J.: A.I.K.E.F.: Adaptive hybrid model for space plasma simulations, Comput. Phys. Commun., 182, 946966, https://doi.org/10.1016/j.cpc.2010.12.033, 2011.

Narita, Y., Glassmeier, K. H., Goldstein, M. L., and Sahraoui, E.: Wave-vector dependence ofmagnetic-turbulence spectra in the solar wind, Phys. Rev. Lett., 104, 171101, https://doi.org/10.1103/PhysRevLett.104.171101, 2010.

Narita, Y.: Four-dimensional energy spectrum for space-time structure of plasma turbulence, Nonlin. Processes Geophys., 21, 4147, https://doi.org/10.5194/npg-21-41-2014, 2014.

Narita, Y., Comisel, H., and Motschmann, U.: Spatial structure of ion-scale plasma turbulence, Front. Phys. 2, 13, https://doi.org/10.3389/fphy.2014.00013, 2014.

Nariyuki, Y., Matsukiyo, S., and Hada, T.: Parametric instabilities of large-amplitude parallel propagating Alfvén waves: 2D PIC simulation, New J. Phys., 10, 8, https://doi.org10.1088/13672630/10/8/083004, 2008.
Shebalin, J. V., Matthaeus, W. H., and Montgomery, D.: Anisotropy in MHD turbulence due to a mean magnetic field, J. Plasma Phys., 29, 525-547, https://doi.org/10.1017/S0022377800000933, 1983.

Viñas, A. F. and Goldstein M. L.: Parametric instabilities of circularly polarized large-amplitude dispersive Alfvén waves: excitation of parallel-propagating electromagnetic daughter waves, J. Plasma Phys., 46, 107, https://doi.org/10.1017/S0022377800015981, 1991a.

Viñas, A. F. and Goldstein M. L.: Parametric instabilities of circularly polarized large-amplitude dispersive Alfvén waves: excitation of obliquely-propagating daughter and side-band waves, J. Plasma Phys., 46, 129, https://doi.org/10.1017/S0022377800015993, 1991 b. 